\title{
Prävention von Typ-2-Diabetes im Rahmen der Gesundheitsstrategie Baden-Württemberg
}

\author{
Eine Einführung
}

Michael Wolfgang Josef Böhme', Günter Karl Kurt Schmolz', Bernhard Otto Böhm²

1 Landesgesundheitsamt Baden-Württemberg im Regierungspräsidium Stuttgart

2 Universitätsklinikum Ulm, Zentrum für Innere Medizin, Klinik für Innere Medizin I

Gesundheit ist eine wichtige Voraussetzung für das Wohlbefinden und die Lebensqualität jedes Einzelnen sowie der gesamten Gesellschaft. Sie ist zudem ein wichtiger Standortfaktor. In diesem Zusammenhang gewinnen chronische lebensstilassoziierte Erkrankungen im Vergleich zu früheren Jahrhunderten zunehmend an Bedeutung. Letzteres liegt zum einen an dem demografischen Wandel hin zu einer immer älteren Bevölkerung sowie an vielfältigen gesundheitlichen Belastungen im Wohnumfeld bzw. der Umwelt und zum anderen an dem „modernen“ Lebensstil. Letzterer ist geprägt durch Bewegungsmangel, ungünstige Ernährung und Stress. Für die Lebensqualität des Einzelnen gewinnen dabei chronische Erkrankungen wie Adipositas, Diabetes, Hypertonie, Fettstoffwechselstörungen, Herz-Kreislauf-Krankheiten, Tumor-Erkrankungen, Rücken- und Gelenkleiden, aber auch Demenz und Depression an Bedeutung. Diese Veränderungen führen insgesamt zu einem kontinuierlichen Wandel der individuellen gesundheitlichen Belange, zu immer neuen Anforderungen an die Gesundheitssysteme hinsichtlich Versorgung aber auch zu ständig zunehmenden Herausforderungen an jeden Einzelnen.

\section{Gesundheitsstrategie Baden-Württem- berg}

Die Stärkung der gesundheitlichen Ressourcen und der Eigenverantwortung jedes Einzelnen, die Schaffung gesunder Lebenswelten sowie eine gemeinsame Vorgehensweise aller im Bereich Gesundheit Aktiven und Verantwortlichen stellen wesentliche Voraussetzungen für eine nachhaltige Bewältigung der aktuellen gesundheitlichen Entwicklungen dar. Diese Herausforderungen wurden 2009 mit der von der Landesregierung beschlossenen Gesundheitsstrategie Baden-Württemberg aufgegriffen, in der grundsätzliche strategische Ziele, Handlungsfelder und Schlüsselbotschaften festgelegt wurden.
Chronische, lebensstilassoziierte Erkrankungen - wie beispielsweise Adipositas, Hypertonie oder Diabetes - sind für das Wohlbefinden und die Lebensqualität jedes Einzelnen sowie der Gesellschaft von zunehmender Bedeutung. Wesentliche Entstehungsfaktoren sind dabei der „moderne“ Lebensstil, der durch Bewegungsmangel, ungünstige Ernährung und Stress geprägt ist, sowie die durchschnittlich immer älter werdende Bevölkerung. Sowohl die Stärkung der gesundheitlichen Ressourcen jedes Einzelnen als auch die Schaffung gesunder Lebenswelten und eine gemeinsame Vorgehensweise aller im Bereich Gesundheit Aktiven und Verantwortlichen stellen wesentliche Voraussetzungen für deren nachhaltige Bewältigung dar. Diese Herausforderungen hat die Gesundheitsstrategie Baden-Württemberg, die 2009 von der Landesregierung beschlossen wurde, aufgegriffen und dabei entsprechende grundsätzliche strategische Ziele, Handlungsfelder und Schlüsselbotschaften festgelegt. Im Rahmen von deren Umsetzung wurde das Gesundheitsziel „Typ-2-Diabetes Risiko senken und Folgen reduzieren “ als eines von 8 Gesundheitszielen mit hoher Priorität vorgeschlagen. Als aktuellen Impuls für eigene oder gemeinsame Aktivitäten für alle thematisch berührten Akteure stellte am 07.10.2011 ein Symposium des Landesgesundheitsamt Baden-Württemberg in Stuttgart zusammenfassend den aktuellen Wissensstand zu Primärprävention und Früherkennung dar und zeigte die Bedeutung der schon bestehenden epidemischen Entwicklung und der Notwendigkeit von Maßnahmen auf.

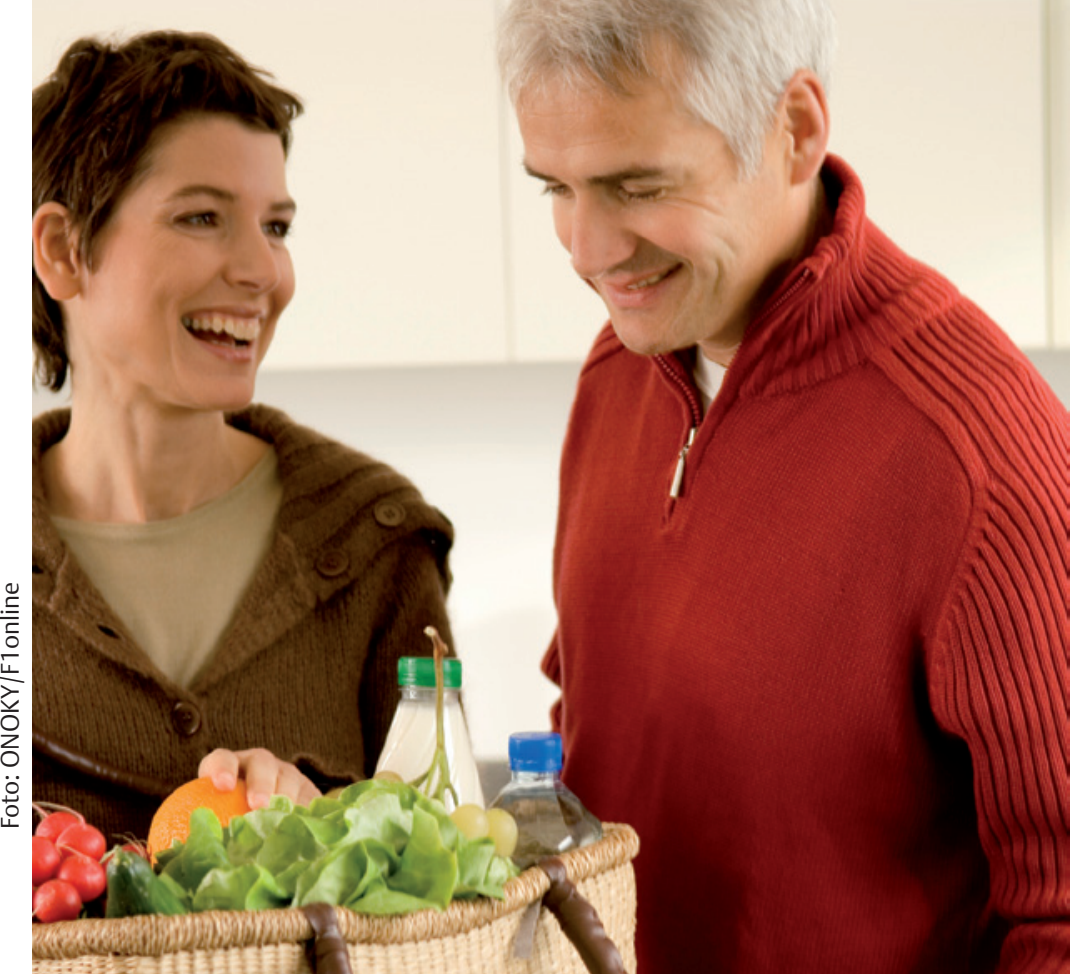


Insgesamt ist die Gesundheitsstrategie BadenWürttemberg dabei darauf ausgerichtet, den Gesundheitszustand der Bevölkerung zu erhalten bzw. zu verbessern und die Wettbewerbsfähigkeit Baden-Württembergs zu stärken. Ein Ziel ist hierbei, lebensstilbedingte chronische Erkrankungen und gesundheitliche Einschränkungen zu vermeiden bzw. ihr Auftreten in eine spätere Lebensphase zu verschieben und chronisch Erkrankte besser zu versorgen. Es wird hierfür insbesondere auf Maßnahmen der Prävention und Gesundheitsförderung gesetzt, die in allen Lebensphasen, Lebenssituationen und Lebenswelten wirksam werden sollen [1].

Eine Voraussetzung für die erfolgreiche Umsetzung von Zielsetzungen der Gesundheitsstrategie Baden-Württemberg ist die Abstimmung gemeinsamer Gesundheitsziele. Hierfür wurde vom Ministerium für Arbeit und Sozialordnung, Familie, Frauen und Senioren Baden-Württemberg eine Expertengruppe aus Vertretern der unterschiedlichsten Bereiche der Gesundheitsversorgung, Gesundheitswirtschaft, Gesundheitsförderung und Prävention eingesetzt. Diese hat 2011 nach einem einjährigen Beratungsprozess unter Berücksichtigung der Schwerpunkte der Gesundheitsstrategie 8 Gesundheitsziele für Baden-Württemberg vorgeschlagen. Bei ihrer Arbeit orientierte sich die Projektgruppe an der Vorgehensweise der bereits erfolgreich durchgeführten Zieleentwicklung auf Bundesebene und den dort erarbeiteten Materialien und Kriterien. Abschließend wurde empfohlen, im Wesentlichen die Bundesziele auch für Baden-Württemberg zu übernehmen, da diese in gleicher Weise auch für Baden-Württemberg von Bedeutung sind, diese jedoch teilweise zu modifizieren bzw. zu ergänzen. Die Gesundheitsziele stellen dabei eine Empfehlung für alle thematisch berührten Akteure dar und sollen eine miteinander abgestimmte Vorgehensweise bei der konkreten weiteren Umsetzung unterstützen. Für letztere ist insbesondere eine vernetzte Vorgehensweise „Vor Ort“ auf kommunaler und regionaler Ebene von entscheidender Bedeutung, wie sie beispielsweise über die sich derzeit im Rahmen der Umsetzung der Gesundheitsstrategie etablierenden kommunalen Gesundheitskonferenzen erfolgen kann.

\section{Gesundheitsziel Typ-2-Diabetes}

„Diabetes mellitus Typ 2 Risiko senken und Folgen reduzieren“ wurde als eines der 8 Ziele mit hoher Priorität vorgeschlagen [2]. Unterstrichen wird die Bedeutung dieses Zieles für BadenWürttemberg z.B. durch die vorliegenden Ergebnisse des derzeit laufenden GEDA-Survey des RKI (Robert-Koch-Institut) aus den Jahren 2008/2009, wonach unter zusätzlicher Berücksichtigung einer Dunkelziffer, wie sie sich aus der KORA-Studie ergibt, etwa jeder Zehnte in Baden-Württemberg von einem Typ-2-Diabetes betroffen ist, etwa jeder Vierte an Bluthochdruck leidet und etwa jeder Zweite übergewichtig oder fettleibig ist. Dieses sind nicht nur weit verbreitete und immer häufiger werdende chronische „Zivilisations-Krankheiten“, sondern sie betreffen zunehmend auch immer jüngere Menschen und können durch Folgekrankheiten die Lebensqualität und Lebenszeit deutlich einschränken. Ein Typ-2-Diabetes verursacht in seiner Frühform häufig über viele Jahre keine oder kaum Beschwerden, kann aber dennoch schon zu Folgekrankheiten geführt haben. In diesem Zusammenhang sind insbesondere auch die Ergebnisse der KORA-Studie von Bedeutung, in der für die Altersgruppe von 55-74 Jahren gezeigt wurde, dass zu den Menschen mit bekanntem Typ-2Diabetes ein erheblicher zusätzlicher fast gleich großer Anteil gefunden werden konnte, der bislang unerkannt war [3-5].

Wichtig für die Empfehlung dieses Gesundheitszieles und dessen weiterer Umsetzung war auch die Tatsache, dass sich ein Typ-2-Diabetes in der Regel durch einen geeigneten Lebensstil positiv beeinflussen lässt, wenn Präventionsangebote frühzeitig beginnen und nachhaltig wahrgenommen werden [6-10]. Bei der zukünftigen Umsetzung des Zieles steht neben der Verbesserung der Versorgung daher insbesondere die Prävention im Vordergrund. Schwerpunkte sind hierbei, auf die bestehenden Risiken hinzuweisen sowie mittels Screening ein bestehendes Risiko früh zu erkennen. Betroffene sollen so die bewusste Entscheidungsmöglichkeit erhalten, unmittelbar für das eigene Wohl aktiv etwas tun zu können, wobei derzeit leider diese Chance auf eine bessere Lebensqualität und auch höhere Lebenserwartung noch von (zu) vielen ,verschenkt" wird.

Eine aktive Gestaltung und Anpassung des eigenen Lebensstils an die „modernen“ Lebensbedingungen ist von Jugend an entscheidend und aus Sicht einer nachhaltigen Prävention dabei vorrangig. Aktuelle Strategien und Programme sollen daher bereits bei den Kindern und Jugendlichen ein zunehmendes Training von Verhaltensweisen hin zu einem Lebensstil mit ausreichender Bewegung und bewusster Ernährung fördern. Hierdurch wird Heranwachsenden die Chance gegeben, diese als selbstverständliche Elemente in den eigenen Lebensstil zu integrieren. Vielen Erwachsenen fehlen allerdings entsprechende bereits in der Kindheit erlernte Verhaltensmuster und Erfahrungen, sodass sie sich die Herausforderungen durch den „modernen“ Lebensstil immer wieder bewusst machen und sich um entsprechende Änderungen ihres Lebensstils aktiv und kontinuierlich bemühen müssen. 
Symposium des Landesgesundheitsamtes Baden-Württemberg 07.10.2011

Das Symposium des Landesgesundheitsamts Baden-Württemberg am 07.10.2011 stellte zusammenfassend als Impuls für eigene oder gemeinsame Aktivitäten von thematisch berührten Akteuren den aktuelle Wissensstand zu Primärprävention und Früherkennung des Metabolischen Syndroms und des Typ-2-Diabetes dar. Es wurde dabei besonders hervorgehoben, dass der moderne Lebensstil mit Bewegungsmangel, ungünstiger Ernährung sowie Stress zwar chronische Volkskrankheiten wie Diabetes und Übergewicht fördert, aber insbesondere auch darauf hingewiesen, dass dieses kein Schicksal sein muss. Durch die verschiedenen Beiträge auf dem Symposium wurde die epidemische Dimension dieser chronischen Krankheiten deutlich aufgezeigt, die zu den vielfältigsten Herausforderungen an den Einzelnen und die Gesellschaft führen. Es wurde betont, dass ein dringender Handlungsbedarf besteht und dass dafür aber auch bereits schon viele Instrumente vorhanden sind, die nur besser, nachhaltiger und vernetzter genutzt werden müssen. Wichtig ist hierbei nicht nur die Suche nach guten Projekten, sondern auch nach geeigneten Partnerschaften und Vernetzung.

Ein besonderes Augenmerk in den WorkshopDiskussionen lag auf dem Ansprechen von speziellen Zielgruppen wie Menschen mit Migrationshintergrund, sozial Benachteiligten und Arbeitslosen, welche häufig nur sehr eingeschränkt erreicht werden. Das Symposium wurde u.a. in Kooperation mit dem Ministerium für Arbeit und Sozialordnung, Familie, Frauen und Senioren Baden-Württemberg, der Deutschen Diabetes-Stiftung (DDS), dem Deutschen Diabetiker Bund, Landesverband Baden-Württemberg und der Arbeitsgemeinschaft Diabetologie Baden-Württemberg (ADBW) durchgeführt. Einige Beiträge des Symposiums werden in der vorliegenden Ausgabe von Diabetes aktuell vorgestellt.

\section{Literatur}

1 Ministerium für Arbeit und Sozialordnung, Familie, Frauen und Senioren: Gesundheitsstrategie BadenWürttemberg. Stuttgart 2009, http://www.sm.badenwuerttemberg.de/fm7/1442/GesundheitsstrategieKonzeption.pdf

2 Ministerium für Arbeit und Sozialordnung, Familie, Frauen und Senioren: AG Standortfaktor Gesundheit - Bericht der Projektgruppe Gesundheitsziele. Stuttgart 2011, http://www.gesundheitsamt-bw. de/SiteCollectionDocuments/10_Kompz_AktivfuerGesundheit/Bericht_Projektgruppe_Gesundheitsziele_08_2011.pdf

3 Robert Koch-Institut. Gesundheit in Deutschland aktuell - Telefonischer Gesundheitssurvey (GEDA). www.gbe-bund.de (21.09.2011).

4 Heidemann C, Du Y, Scheidt-Nave C. Diabetes mellitus in Deutschland. Hrsg. Robert Koch-Institut Berlin 2011, GBE kompakt www.rki.de/gbe-kompakt (Stand 06.05.2011)
Prevention of type 2 diabetes within the framework of the health strategy of Baden-Wuerttemberg

Chronic, lifestyle-associated diseases such as obesity, hypertension or diabetes become increasingly important for the well-being and quality of life of individuals as well as the society. Significant factors for their emergence are the ,modern' lifestyle, which is characterized by a lack of exercise, unhealthy diet and stress, and the on average increasing age of the population. Both the strengthening of the health resources of each individual and the creation of a healthy living environment and the common action of all stakeholders in the health sector are important for the durable overcoming of these diseases. The health strategy of Baden-Wuerttemberg, adopted by the federal state government in 2009, has taken up this challenge and addressed basic strategical objectives, fields of action, and key messages. In the context of the further implementation, the health target to ,reduce the risk and outcome of type 2 diabetes', was suggested with high priority to be one of eight health goals. In this connection, the actual state of knowledge on prevention and early diagnosis of diabetes was presented at a symposium of the Baden-Wuerttemberg state health office, held in Stuttgart on October 7, 2011, as an impulse for individual or common activities. Thereby, the importance of the ongoing epidemic development of diabetes and the urgency of measures were pointed out.

\section{Key words}

diabetes - prevention - health goal - lifestyle - health strategy

5 Rathmann W, Haastert B, Icks A et al. High prevalence of undiagnosed diabetes mellitus in Southern Germany: target populations for efficient screening. The KORA survey 2000. Diabetologia 2003; 46: 182189

6 Tuomilehto J, Lindström J, Eriksson JG et al. Prevention of type 2 diabetes mellitus by changes in lifestyle among subjects with impaired glucose tolerance. N Engl J Med 2001; 344: 1343-1350

7 Knowler WC, Barrett-Conner E, Fowler SE et al. Diabetes Prevention Program research Group. Reduction in the incidence of type 2 diabetes with lifestyle intervention or metformin. N Engl J Med 2002; 346: 393-403

8 Gillies CL, Abrams KR, Lambert PC et al. Pharmacological and lifestyle interventions to prevent or delay type 2 diabetes in people with impaired glucose tolerance: systematic review and meta-analysis. BM] 2007; 334: 299-308

9 Knowler WC, Fowler SE, Hamman RF et al. Diabetes Prevention Program Research Group. 10-year followup of diabetes incidence and weight loss in the Diabetes Prevention Program Outcomes Study. Lancet 2009; 374: 1677-1686

10 Lim E.L, Hollingeworth KG, Aribisala BS, Chen M], Mathers JC, Taylor R. Reversal of type 2 diabetes: normalisation of beta cell function in association with decreased pancreas and liver triacylglycerol. Diabetologia 2011; 54: 2506-2514

\section{Korrespondenz}

Prof. Dr. M. Böhme

Landesgesundheitsamt Baden-Württemberg

im Regierungspräsidium Stuttgart

Nordbahnhofstr. 135

70191 Stuttgart

\section{Autorenerklärung}

Die Autoren erklären, dass für diesen Artikel

keine Interessenkonflikte bestehen. 